\title{
Radiative corrections to the magnetic moments of the proton and the neutron 1
}

\author{
N. Kaiser \\ Physik-Department, Technische Universität München, D-85747 Garching, Germany \\ email:nkaiser@ph.tum.de
}

\begin{abstract}
We estimate the radiative corrections of order $\alpha / \pi$ to the magnetic moments of the proton and the neutron. The photon-loop diagram of the vertex-correction type is evaluated with phenomenological nucleon vector form factors. Infrared-finiteness and gauge-invariance require the inclusion of the wave-function renormalization factor from the self-energy diagram. Using recent empirical form factor parametrizations the corrections amount to $\delta \kappa_{p}=-3.42 \cdot 10^{-3}$ and $\delta \kappa_{n}=1.34 \cdot 10^{-3}$. We study also the effects from photon-loops with internal $\Delta(1232)$-isobars. For two customary versions of the $\Delta N \gamma$-vertex and spin-3/2 propagator, these radiative corrections have values of $\delta \kappa_{p}^{(\Delta)}=(-0.9,0.0) \cdot 10^{-3}$ and $\delta \kappa_{n}^{(\Delta)}=(1.2,-0.8) \cdot 10^{-3}$, respectively.
\end{abstract}

\section{Introduction}

The magnetic moments of the nucleon (proton and neutron) are important structure constants and their understanding represents a challenge for any model or theory of the strong interactions. The measured values of the proton magnetic moment $\mu_{p}=1+\kappa_{p}=2.792847356(23)$ and the neutron magnetic moment $\mu_{n}=0+\kappa_{n}=-1.9130427(5)$, both given in units of the nuclear magneton $e \hbar / 2 M_{p}$, are extremely precise [1]. The large anomalous magnetic moments $\kappa_{p}$ and $\kappa_{n}$, describing the deviations from the values 1 and 0 of a charged and neutral Dirac-particle, arise predominantely from the strong interactions in these composite three-quark systems. However, at the given experimental precision of $\kappa_{p}$ and $\kappa_{n}$, their full-fledged values cannot be attributed to the strong interactions alone, and electromagnetic effects (or radiative corrections) play also a substantial role. In the case of charged leptons a leading correction of $\delta \kappa_{\ell}=\alpha / 2 \pi=1.1614 \cdot 10^{-3}$ is provided by the one-photon loop diagrams, calculated first by J. Schwinger in 1948. Due to the ubiquitous prefactor $\alpha / \pi$, one can expect that radiative corrections to the nucleon magnetic moments will similarly amount in magnitude to a few per mille. However, when deriving the analogous radiative corrections for nucleons one has to take into account that these composite objects possess an electromagnetic structure, preformed by the strong interaction, and that they are excitable into baryonic resonances. The purpose of this paper is to provide quantitative estimates for the radiative corrections to the nucleon magnetic moments based on parametrized nucleon form factors and a Lorentz-covariant treatment of the low-lying spin-3/2 $\Delta(1232)$-resonance.

The present paper is organized as follows. In section 3 we calculate the one-photon loop diagrams of the vertex-correction and self-energy type with inclusion of phenomenological nucleon vector form factors. As prepared in section 2, the radiative correction to the anomalous magnetic moment is extracted by a projection technique and after Wick-rotation to euclidean momentumspace and angular integration one obtains a set of weight-functions. These serve to express the quantity of interest, $\delta \kappa=F_{2}^{(\gamma)}(0)$, as a one-parameter integral over a quadratic form in the nucleon vector form factors. Moreover, we verify that the inclusion of the wave-function renormalization

\footnotetext{
${ }^{1}$ This work has been supported in part by DFG and NSFC (CRC110).
} 
factor $Z_{2}$ from the (electromagnetic) self-energy diagram leads to an infrared-finite and gaugeinvariant result. Several parametrizations of the proton and neutron vector form factors are used to evaluate the integral-represention of $\delta \kappa_{p}$ and $\delta \kappa_{n}$. Section 4 is devoted to the calculation of the photon-loop diagrams with a single intermediate $\Delta(1232)$-isobar. We employ common forms of the Rarita-Schwinger propagator and the $\Delta N \gamma$-vertex amended by a phenomenological transition form factor. Section 5 deals with the mechanism, where a charged $\Delta^{+}$-isobar propagates further inside the photon-loop after electromagnetic interaction, which is applicable only to the proton. The pertinent $\Delta^{+} \Delta^{+} \gamma$-vertex is obtained by gauging the kinetic term of the free Rarita-Schwinger Lagrangian. In section 6 we study alternative forms of the Rarita-Schwinger propagator and the $\Delta N \gamma$-vertex, which incorporate the feature of projecting onto the spin-3/2 degrees of freedom. The results obtained with both Lorentz-covariant treatments of the $\Delta(1232)$-isobar may provide a range for the size of baryonic resonance contributions to the radiative corrections to the nucleon magnetic moments. Finally, section 7 ends with a short summary and in the appendix expressions for the electromagnetic mass shifts are collected and discussed.

\section{Preparation}

Let us start with recalling the form of the on-shell vector-vertex for a spin- $1 / 2$ nucleon of (average) mass $M=939 \mathrm{MeV}$. Imposing current-conservation and the usual discrete symmetries, the transition matrix-element $N\left(p_{1}\right)+\gamma^{*}(q) \rightarrow N\left(p_{2}\right)$ reads:

$$
\Gamma^{\mu}=\gamma^{\mu} F_{1}^{(\gamma)}(-t)+\frac{i}{2 M} \sigma^{\mu \nu} q_{\nu} F_{2}^{(\gamma)}(-t)
$$

with the spin-tensor $\sigma^{\mu \nu}=i\left(\gamma^{\mu} \gamma^{\nu}-\gamma^{\nu} \gamma^{\mu}\right) / 2$ and the squared momentum-transfer $t=q^{2}=\left(p_{2}-\right.$ $\left.p_{1}\right)^{2} \leq 0$. The superscript $(\gamma)$ on the Dirac and Pauli form factors $F_{1,2}^{(\gamma)}(-t)$ should indicate that $\Gamma^{\mu}$ arises from a photon-loop diagram. In order to extract the radiative correction to the magnetic moment, one has to project out first the loop-induced Pauli form factor $F_{2}^{(\gamma)}(-t)$. This is achieved by multiplying $\Gamma^{\mu}$ with a suitable projection operator [2] and taking the Dirac-trace:

$$
F_{2}^{(\gamma)}(-t)=\frac{M}{t\left(4 M^{2}-t\right)} \operatorname{Tr}\left\{\Gamma^{\mu}\left(\gamma \cdot p_{1}+M\right)\left[M \gamma_{\mu}+\frac{t+2 M^{2}}{t-4 M^{2}}\left(p_{1}+p_{2}\right)_{\mu}\right]\left(\gamma \cdot p_{2}+M\right)\right\}
$$

In the next step the limit $t \rightarrow 0$ has to be performed with care before the four-dimensional integration over the loop-momentum $l$. By choosing the Breit-frame with $q_{0}=0$ and averaging over the directions of the (small) momentum-transfer $\vec{q}$, one can effectively make the substitution: $(l \cdot q)^{2}=(\vec{l} \cdot \vec{q})^{2} \rightarrow \vec{l}^{2} \vec{q}^{2} / 3=-\vec{l}^{2} t / 3$. During this procedure the momentum-sum $p_{1}+p_{2}=$ $\left(\sqrt{4 M^{2}+\vec{q}^{2}}, \overrightarrow{0}\right)$ is kept fixed. After setting $t=0$, the resulting loop-integrand depends only on $l^{2}$ and $l \cdot p_{1,2}=M l_{0}$. One performs a Wick-rotation to euclidean momentum-space, $l_{0}=i l_{4}$, and sets $l_{4}=\ell z$ with $z=\cos \theta$ the cosine of the polar angle $\theta$ in four dimensions. By combining these steps, a loop-integral including the factor $-1 / l^{2}$ from a photon-propagator gets reduced to:

$$
\int \frac{d^{4} l}{(2 \pi)^{4} i} \frac{1}{\left(-l^{2}\right)}[\ldots]=\frac{M^{2}}{4 \pi^{3}} \int_{0}^{\infty} d x \int_{-1}^{1} d z x \sqrt{1-z^{2}}[\ldots],
$$

where one has set the euclidean length to $\ell=x M$. The remaining terms $[\ldots]$ of the loop-integrand depend rationally on $z$, such that the angular integral can always be performed analytically. The outcome of the loop-calculation at this stage are $x$-dependent weight-functions.

In the following sections we apply these techniques to calculate the radiative corrections to the nucleon magnetic moments as they arise from photon-loop diagrams involving nucleons and $\Delta(1232)$-isobars. The pertinent electromagnetic vertices are supplemented by phenomenological form factors which should represent the (internal) baryon structure. 


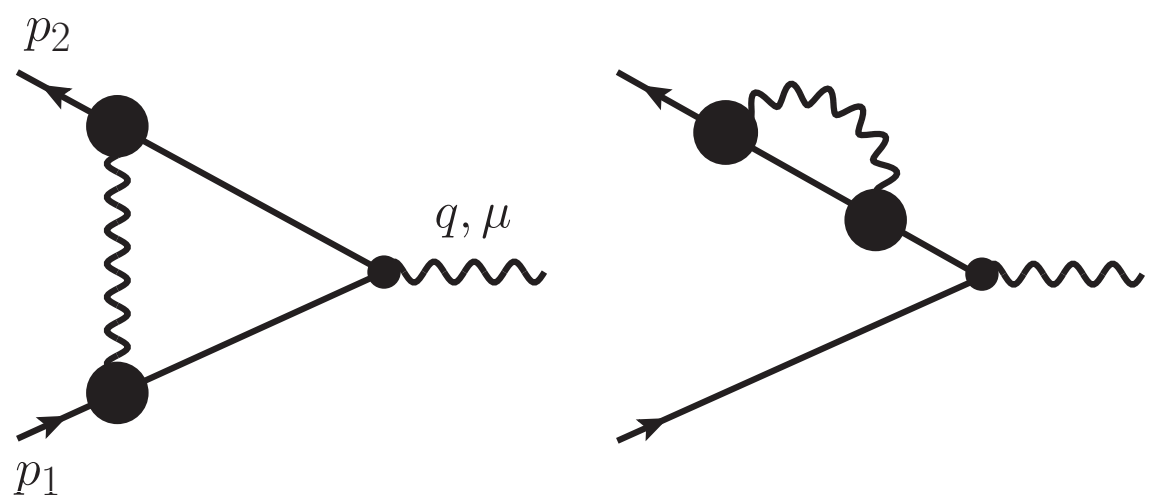

Figure 1: Left: Triangular photon-loop diagram with intermediate nucleons. Right: Electromagnetic self-energy correction on an external nucleon line. The corresponding mirror diagram is not shown.

\section{Photon-loop diagrams with nucleons}

The triangular photon-loop diagram with intermediate nucleons is shown on the left in Fig. 1. We evaluate it with three (off-shell) vector-vertices of the form $\Gamma^{\mu}$ in eq.(1), where the form factors $F_{1,2}(\ell)$ and $F_{1,2}(0)$ refer to the nucleon structure arising from strong interactions. Carrying out the calculational steps as outlined in section 2, one arrives at the following (partial) result for the radiative correction to the nucleon magnetic moments:

$$
\begin{aligned}
F_{2}^{(\gamma)}(0)= & \frac{\alpha}{\pi} \int_{0}^{\infty} d x\left\{\left[\left(\frac{2+4 x^{2}+x^{4}}{\sqrt{4+x^{2}}}-2 x-x^{3}\right) c+\left(\frac{3+x^{2}}{\sqrt{4+x^{2}}}-x-\frac{1}{x}\right) \kappa\right] F_{1}^{2}(x M)\right. \\
& +\left[\left(\frac{x^{2}\left(7+2 x^{2}\right)}{\sqrt{4+x^{2}}}-3 x-2 x^{3}\right) c+\left(2 x+3 x^{3}-\frac{x^{2}\left(8+3 x^{2}\right)}{\sqrt{4+x^{2}}}\right) \frac{\kappa}{8}\right] F_{1}(x M) F_{2}(x M) \\
& +\left[\left(\frac{x^{2}}{\sqrt{4+x^{2}}}\left(16+x^{2}-x^{4}\right)-8 x-3 x^{3}+x^{5}\right) \frac{c}{8}\right. \\
& \left.\left.+\left(\frac{x^{2}}{\sqrt{4+x^{2}}}\left(8+5 x^{2}+x^{4}\right)-4 x-3 x^{3}-x^{5}\right) \frac{\kappa}{16}\right] F_{2}^{2}(x M)\right\} .
\end{aligned}
$$

Here, $c=1,0$ denote the charge and $\kappa=1.793,-1.913$ the anomalous magnetic moment of the proton and neutron, respectively. Of course, in the end the third decimal place of $\kappa_{p, n}$ should be changed in order to treat properly only the strong interaction part. $\alpha=e^{2} / 4 \pi=1 / 137.036$ denotes the usual fine-structure constant. It should be noted that the triangle diagram has been evaluated with a photon-propagator $-i g^{\mu \nu} / l^{2}$ in Feynman gauge. The integral $\int_{0}^{\infty} d x$ over the first weight-function $\left(2+4 x^{2}+x^{4}\right) / \sqrt{4+x^{2}}-2 x-x^{3}$ has the value $1 / 2$, and this way one recovers the famous result $F_{2}^{(\gamma)}(0)=\alpha / 2 \pi$ of Schwinger for a structureless spin-1/2 Dirac-particle.

The partial result in eq.(4) has an obvious problem in the form of the infrared-singular $1 / x$-term in the weight-function multiplying $\kappa F_{1}^{2}(x M)$. The only other diagrams available at order $\alpha$ are those with a self-energy correction on an external nucleon line, shown on the right in Fig. 1. Their effect is to multiply the tree-level vector-vertex with the nucleon wave-function renormalization factor $Z_{2}$. In order to extract this quantity, one decomposes the Dirac self-energy $\Sigma$ into a scalar and a vector part: $\Sigma=\Sigma_{s}\left(p^{2}\right)+\gamma \cdot p \Sigma_{v}\left(p^{2}\right)$. By setting thereafter $p=\left(p_{0}, \overrightarrow{0}\right)$ the wave-function renormalization factor $Z_{2}$ is obtained as the derivative of $\Sigma_{s}\left(p_{0}^{2}\right)+p_{0} \Sigma_{v}\left(p_{0}^{2}\right)$ with respect $p_{0}$ at $p_{0}=M$. The electromagnetic mass-shift follows in a simpler way as: $\delta M=\Sigma_{s}\left(M^{2}\right)+M \Sigma_{v}\left(M^{2}\right)$. After some calculation one arrives at the following expression for the wave-function renormalization 
factor $Z_{2}$ from the self-energy subdiagram in Fig. 1:

$$
\begin{aligned}
Z_{2}= & \frac{3 \alpha}{4 \pi} \int_{0}^{\infty} d x\left\{\left[\frac{4}{3 x}-x^{3}+\frac{x^{4}+2 x^{2}-4}{\sqrt{4+x^{2}}}\right] F_{1}^{2}(x M)\right. \\
& +\left[\frac{x^{2}\left(16+5 x^{2}\right)}{2 \sqrt{4+x^{2}}}-3 x-\frac{5 x^{3}}{2}\right] F_{1}(x M) F_{2}(x M) \\
& \left.+\left[\frac{x^{2}}{8 \sqrt{4+x^{2}}}\left(16+2 x^{2}-x^{4}\right)-x-\frac{x^{3}}{2}+\frac{x^{5}}{8}\right] F_{2}^{2}(x M)\right\} .
\end{aligned}
$$

One observes that in the combination $F_{2}^{(\gamma)}(0)+Z_{2} \kappa$ the singular $1 / x$-term drops out, and thus an infrared-finite radiative correction is achieved. Moreover, one has to check gauge-invariance by working with a general photon-propagator:

$$
\frac{i}{-l^{2}}\left(g^{\mu \nu}+\xi \frac{l^{\mu} l^{\nu}}{-l^{2}}\right) .
$$

Its $\xi$-dependent longitudinal part produces via the triangular photon-loop diagram a contribution:

$$
F_{2}^{(\gamma)}(0)=-\frac{\alpha \xi}{2 \pi} \kappa \int_{0}^{\infty} d x x^{-1} F_{1}^{2}(x M)
$$

which gets exactly canceled by a contribution to the $Z_{2}$-factor from the self-energy subdiagram:

$$
Z_{2}=\frac{\alpha \xi}{2 \pi} \int_{0}^{\infty} d x x^{-1} F_{1}^{2}(x M)
$$

The infrared-finite and gauge-invariant total result $F_{2}^{(\gamma)}(0)+Z_{2} \kappa$ from the photon-loop diagrams of vertex-correction and self-energy type in Fig. 1 reads finally:

$$
\begin{aligned}
F_{2}^{(\gamma)}(0)= & \frac{\alpha}{\pi} \int_{0}^{\infty} d x\left\{\left[\left(\frac{2+4 x^{2}+x^{4}}{\sqrt{4+x^{2}}}-2 x-x^{3}\right) c+\left(\frac{x^{2}\left(10+3 x^{2}\right)}{\sqrt{4+x^{2}}}-4 x-3 x^{3}\right) \frac{\kappa}{4}\right] F_{1}^{2}(x M)\right. \\
& +\left[\left(\frac{x^{2}\left(7+2 x^{2}\right)}{\sqrt{4+x^{2}}}-3 x-2 x^{3}\right) c+\left(\frac{x^{2}\left(10+3 x^{2}\right)}{\sqrt{4+x^{2}}}-4 x-3 x^{3}\right) \frac{\kappa}{2}\right] F_{1}(x M) F_{2}(x M) \\
& +\left[\left(\frac{x^{2}}{\sqrt{4+x^{2}}}\left(16+x^{2}-x^{4}\right)-8 x-3 x^{3}+x^{5}\right) \frac{c}{8}\right. \\
& \left.\left.+\left(\frac{x^{2}}{\sqrt{4+x^{2}}}\left(64+16 x^{2}-x^{4}\right)-32 x-18 x^{3}+x^{5}\right) \frac{\kappa}{32}\right] F_{2}^{2}(x M)\right\},
\end{aligned}
$$

with $c=1,0$ the charges and $\kappa=1.793,-1.913$ the anomalous magnetic moments of nucleons.

The numerical evaluation of the integral-formula in eq.(9) for the radiative correction to the nucleon magnetic moments requires as input the momentum-dependent form factors $F_{1,2}(Q)$. The simplest $Q$-dependence, which roughly reproduces the trend of the data, is a dipole parametrization of the electric and magnetic form factor [3]:

$$
\begin{gathered}
G_{E}(Q)=F_{1}(Q)-\frac{Q^{2}}{4 M^{2}} F_{2}(Q)=c\left(1+\frac{Q^{2}}{\Lambda^{2}}\right)^{-2} \\
G_{M}(Q)=F_{1}(Q)+F_{2}(Q)=(c+\kappa)\left(1+\frac{Q^{2}}{\Lambda^{2}}\right)^{-2}
\end{gathered}
$$




\begin{tabular}{|c|ccc|}
\hline form factor & dipole & DRA & Ina \\
\hline $10^{3} \cdot \delta \kappa_{p}$ & -3.466 & -3.495 & -3.423 \\
$10^{3} \cdot \delta \kappa_{n}$ & 1.370 & 1.336 & 1.337 \\
\hline
\end{tabular}

Tab.1: Numerical values of radiative corrections to the proton and neutron magnetic moments.

with $\Lambda=843 \mathrm{MeV}$ the empirical dipole mass. More elaborate parametrizations of the nucleon form factors are obtained in the dispersion relation analysis (DRA) [4. In addition to the data from elastic electron-scattering, fits based on this method include the constraints from unitarity, analyticity and crossing symmetry as well as the QCD-asymptotics. We employ here the expressions for the isoscalar and isovector form factors $F_{1,2}^{(s, v)}(Q)$ given in appendix $\mathrm{C}$ of ref. [4]. Moreover, improved fits of the nucleon vector form factors have been obtained recently in refs. [5, 6, 7] by including two-photon exchange corrections in the analysis of all available and updated elastic electron-proton scattering data. The fitted results for $F_{1,2}^{(s, v)}(Q)$ are presented in an analytical form as sums of vector-boson poles supplemented by rational parametrizations of the contributions from the isovectorial $\pi \pi$-continuum [8].

In Table 1 numerical values for the radiative correction $\delta \kappa_{p, n}=F_{2}^{(\gamma)}(0)$ to the proton/neutron magnetic moment are listed. The three columns refer to the dipole parametrization (dipole), the earlier fit [4] using dispersion relations (DRA), and the most recent fit [8] including $2 \gamma$-exchange corrections (Ina). Modulo some slight variations, the radiative correction $\delta \kappa_{p} \simeq-3.4 \cdot 10^{-3}$ to the proton magnetic moment is in magnitude about 2.5 times larger than $\delta \kappa_{n} \simeq 1.3 \cdot 10^{-3}$ for the neutron. In both cases the radiative corrections amount to about $-1 \%$ of the empirical magnetic moments $1+\kappa_{p}=2.793$ and $\kappa_{n}=-1.913$. In order to illustrate how the numerical values in Table 1 have come about, we present in Fig. 2 the integrand for $\delta \kappa_{p}$, without the small prefactor $\alpha / \pi$. One observes distributions that take on negative values for $x>0.05$ and peak around $x=0.3$, corresponding to a momentum-transfer of $Q=\Lambda / 3$ (with $\Lambda=843 \mathrm{MeV}$ the dipole mass). The fast transition from positive to negative values has its origin in the six $x$-dependent weight-functions entering eq.(9), where all exept the first one are negative functions. The upper full curve in Fig. 2 (with total area $1 / 2$ under it) shows the different situation for the radiative correction to the magnetic moment of a (structureless) lepton. Likewise, the integrand for $\delta \kappa_{n}$ (without the factor $\alpha / \pi)$ is displayed in Fig. 3. In this case essentially only the term in the last line of eq.(9) contributes. Since a negative weight-function gets multiplied by $\kappa_{n}=-1.913$, one obtains a positive integrand and a positive radiative correction of $\delta \kappa_{n} \simeq 1.3 \cdot 10^{-3}$.

At this point one can draw the interesting conclusion that the large anomalous magnetic moments $\kappa_{p, n}$ of nucleons, predetermined by the strong interaction, change the sign of the leading radiative correction proportional to $\alpha / \pi$ in comparison to pointlike leptons.

\section{Photon-loop diagrams with nucleon and delta-isobar}

In the following sections we study effects of the $\Delta(1232)$-resonance on radiative corrections to the nucleon magnetic moments. The $\Delta(1232)$-isobar with isospin-3/2 and spin- $3 / 2$ is the lowestlying and most prominent excitation of the nucleon and the charge-states $\left(\Delta^{+}, \Delta^{0}\right)$ decay also electromagnetically into $(p \gamma, n \gamma)$ final-states. The pertinent electromagnetic transition matrixelements are related to measurable helicity amplitudes [3]. The present calculation of photonloop diagrams requires yet a Lorentz-covariant formulation in which the spin-3/2 delta-isobar is described by a Rarita-Schwinger spinor $\Psi_{\alpha}$. There exists an extensive literature [9, 10, 11] about phenomenological applications as well as potential conceptual problems of the Rarita-Schwinger formalism employed for the $\Delta(1232)$-resonance. A commonly used (minimal) form of the $\Delta N \gamma$ - 


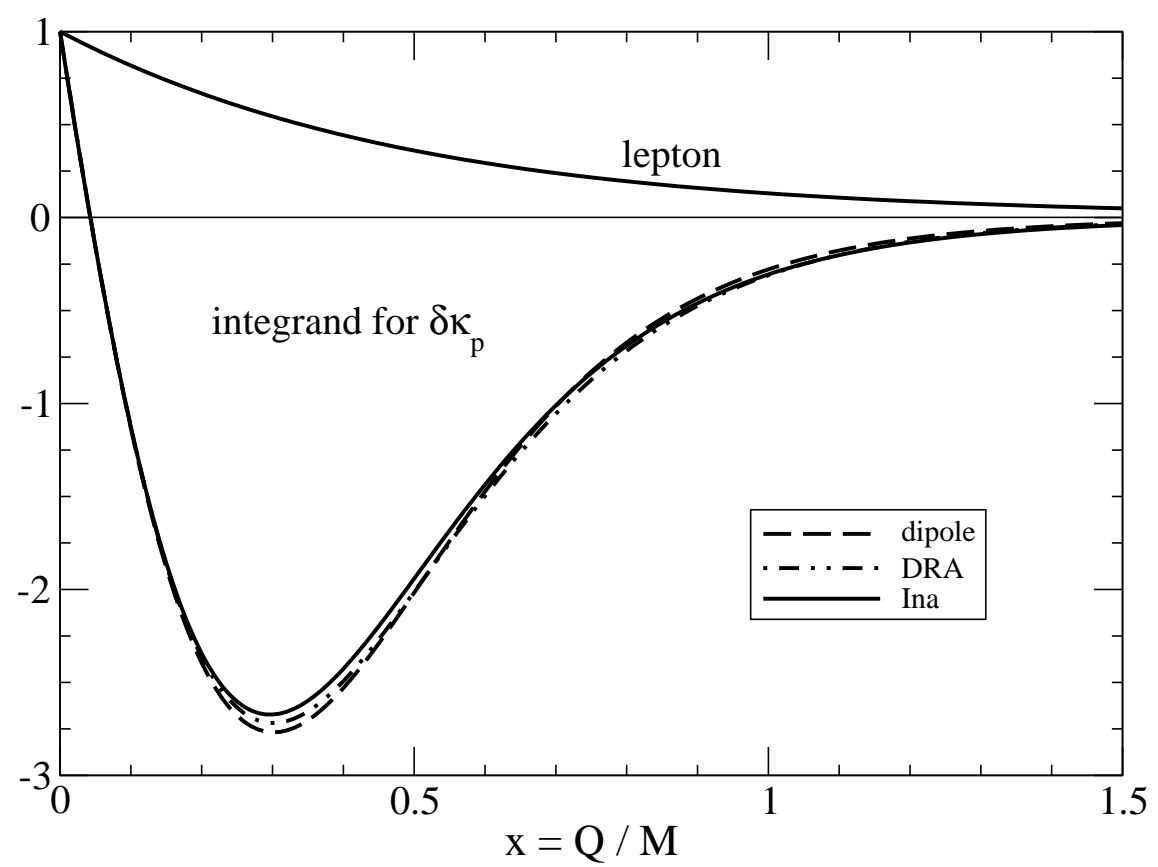

Figure 2: Integrand (without factor $\alpha / \pi$ ) for the radiative correction to the proton magnetic moment.

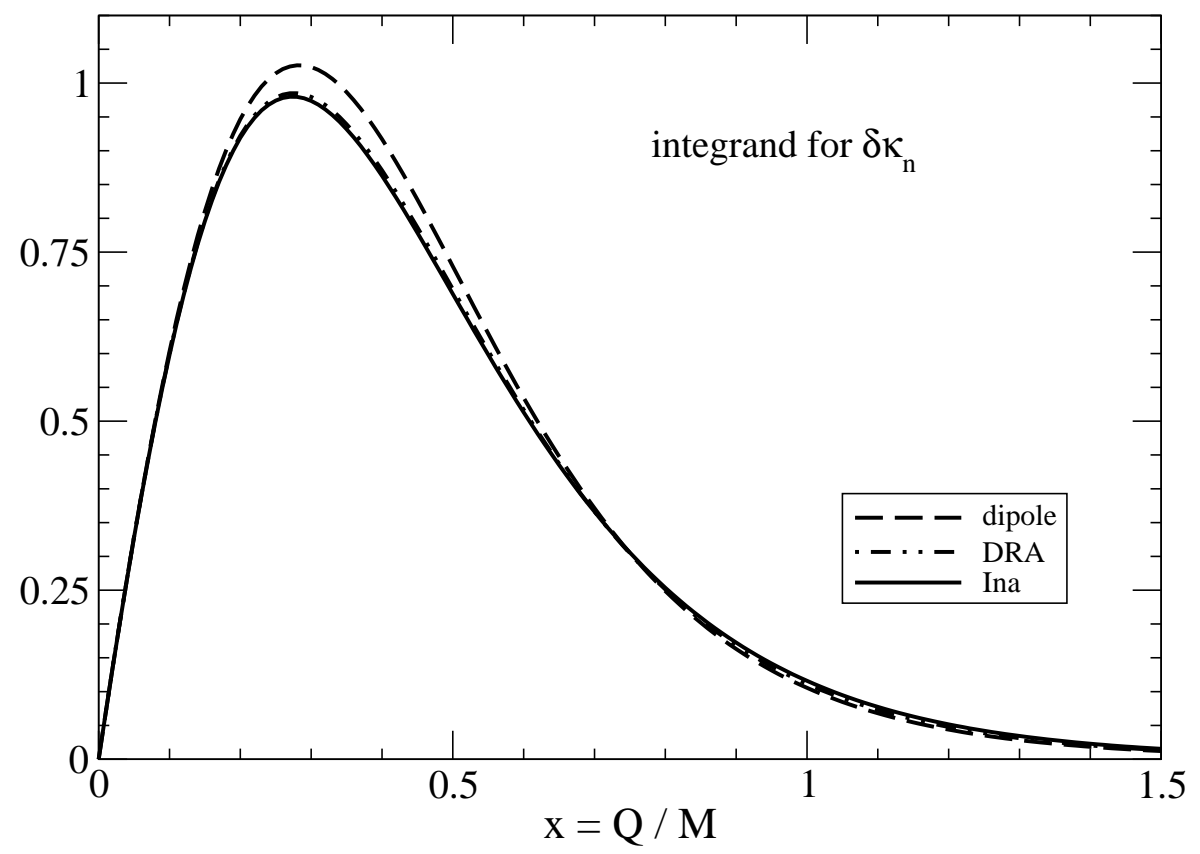

Figure 3: Integrand (without factor $\alpha / \pi$ ) for the radiative correction to the neutron magnetic moment.

vertex reads [9, 12]:

$$
V_{1}^{\mu \alpha}=\frac{i e \kappa^{*}}{\sqrt{6} M}\left(g^{\mu \alpha} \gamma \cdot l-\gamma^{\mu} l^{\alpha}\right) \gamma_{5} G_{\Delta}\left(\sqrt{-l^{2}}\right),
$$

with $\mu$ the vector-index to couple the virtual photon with incoming four-momentum $l$. The stated vertex includes (in the prefactor) an isospin Clebsch-Gordan coefficient $\left\langle\frac{3}{2} \frac{ \pm 1}{2} \mid 10, \frac{1}{2} \frac{ \pm 1}{2}\right\rangle=\sqrt{2 / 3}$ and a phenomenological transition form factor $G_{\Delta}\left(\sqrt{-l^{2}}\right)$, normalized to $G_{\Delta}(0)=1$. For the $\Delta \rightarrow N \gamma$ transition magnetic moment $\kappa^{*}$ we choose the large- $N_{c}$ value $\kappa^{*}=3 \sqrt{2}\left(1+\kappa_{p}-\kappa_{n}\right) / 4 \simeq 5.0$, which agrees well with empirical determinations [9]. Note that the off-shell vertex in eq.(12) is gauge- 


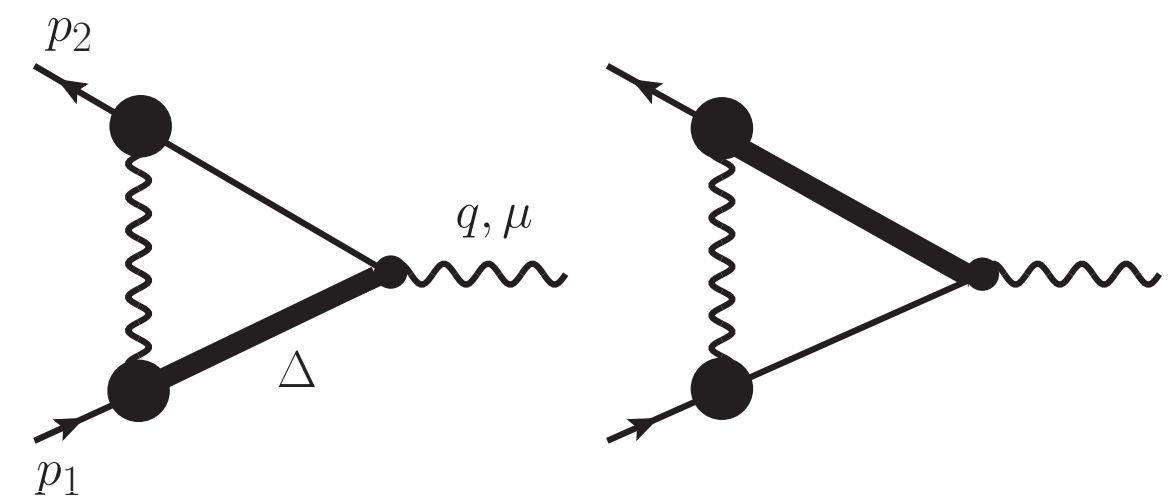

Figure 4: Triangular photon-loop diagrams with intermediate nucleon und delta-isobar. Both diagrams contribute equally.

invariant be construction, $l_{\mu} V_{1}^{\mu \alpha}=0$, and therefore the $\xi$-dependent part of the photon-propagator in eq.(6) will drop out immediately in our loop-calculation. Moreover, it should be mentioned that extensions of the vertex $V_{1}^{\mu \alpha}$ with further coupling constants and off-shell parameters have been proposed [9, 12], but these parameters are not well determined. Another relevant ingredient for our loop-calculation is the Rarita-Schwinger propagator, whose common form reads [9, 12]:

$$
\frac{i}{3} \frac{\gamma \cdot P+M_{\Delta}}{M_{\Delta}^{2}-P^{2}}\left(3 g_{\alpha \beta}-\gamma_{\alpha} \gamma_{\beta}-\frac{2 P_{\alpha} P_{\beta}}{M_{\Delta}^{2}}+\frac{P_{\alpha} \gamma_{\beta}-\gamma_{\alpha} P_{\beta}}{M_{\Delta}}\right),
$$

with $P$ the four-momentum of the delta-isobar propagating from index $\beta$ to index $\alpha$.

Fig. 4 shows the two triangular photon-loop diagrams where one internal nucleon is excited (electromagnetically) into a $\Delta$ (1232)-isobar. Since both diagrams are mirror partners of each other they contribute equally to the on-shell vector-vertex $\Gamma^{\mu}$. Carrying out the necessary calculations, one arrives at the following contribution from the photon-loop diagrams in Fig. 4 to the radiatively induced magnetic moments of nucleons:

$$
\begin{aligned}
F_{2}^{(\gamma)}(0)= & \frac{\alpha \kappa^{* 2}}{54 \pi r^{2}} \int_{0}^{\infty} d x G_{\Delta}(x M)\left\{F _ { 1 } ( x M ) \left[\frac{2}{x}\left(r^{2}-1\right)^{2}\left(r^{2}-r-2 r^{3}\right)-6 x\left(r+r^{3}+2 r^{5}\right)\right.\right. \\
& -6 x^{3}\left(2+r+r^{2}+2 r^{3}\right)-x^{5}(2+r)+\frac{x^{2}}{r^{2}-1} \sqrt{4+x^{2}}\left(4 r^{2}-8-6 r-6 r^{3}\right. \\
& \left.-x^{2}\left(2+3 r+2 r^{2}+3 r^{3}\right)\right)+2 r \sqrt{\left(r^{2}-1+x^{2}\right)^{2}+4 x^{2}}\left(\frac{r^{2}-1}{x}\left(1-r+2 r^{2}\right)\right. \\
& \left.\left.+\frac{x}{r^{2}-1}\left(2+r+r^{3}+4 r^{4}+\left(1+2 r+2 r^{2}\right) x^{2}\right)\right)\right] \\
& +F_{2}(x M)\left[-\frac{r}{2 x}\left(r^{2}-1\right)^{2}\left(1+4 r+3 r^{2}\right)-x\left(r+9 r^{2}+4 r^{3}+3 r^{4}+7 r^{5}\right)\right. \\
& -3 x^{3} r^{2}(2+3 r)+\frac{x^{5}}{2}\left(6+r+x^{2}\right)+\frac{x^{2}}{r^{2}-1} \sqrt{4+x^{2}}\left(-8 r^{2}(1+r)\right. \\
& \left.+\frac{x^{2}}{2}\left(4+r-14 r^{2}-7 r^{3}+\left(1+r-r^{2}\right) x^{2}\right)\right)+\frac{r}{2} \sqrt{\left(r^{2}-1+x^{2}\right)^{2}+4 x^{2}}\left(\frac{r^{2}-1}{x}\right. \\
& \left.\left.\left.\times\left(1+4 r+3 r^{2}\right)+\frac{x}{r-1}\left(1+13 r-9 r^{2}+11 r^{3}\right)+\frac{x^{3}}{r^{2}-1}\left(7 r^{2}+10 r-1-x^{2}\right)\right)\right]\right\},
\end{aligned}
$$




\begin{tabular}{|c|ccc|}
\hline form factor & dipole & DRA & Ina \\
\hline $10^{3} \cdot \delta \kappa_{p}$ & -2.705 & -2.750 & -2.695 \\
$10^{3} \cdot \delta \kappa_{n}$ & 2.367 & 2.396 & 2.412 \\
\hline
\end{tabular}

Tab.2: Radiative corrections to the proton and neutron magnetic moments arising from $\Delta N \gamma$ triangle-diagrams.

with $r=M_{\Delta} / M=1.312$ the ratio between the delta mass and the nucleon mass. One should note that the (lengthy) weight-functions multiplying $F_{1,2}(x M)$ behave near $x=0$ as order $x^{2}$. In order to evaluate the integral in eq.(14) numerically we use for the transition form factor $G_{\Delta}(x M)$ a dipole multiplied by an exponential function:

$$
G_{\Delta}(Q)=\left(1+\frac{Q^{2}}{\Lambda^{2}}\right)^{-2} \exp \left(-\frac{Q^{2}}{7 \Lambda^{2}}\right) .
$$

Such an approximate $Q$-dependence has been extracted in ref.[13] from pion-electroproduction data in the $\Delta(1232)$-resonance region.

Table 2 gives the numerical values for $\delta \kappa_{p}$ and $\delta \kappa_{p}$ from the $\Delta N \gamma$ loop-diagrams for the three choices (dipole, DRA, Ina) of the nucleon vector form factors $F_{1,2}(x M)$. One obtains sizeable contributions of $\delta \kappa_{p} \simeq-2.7 \cdot 10^{-3}$ and $\delta \kappa_{n} \simeq 2.4 \cdot 10^{-3}$ with the same sign as those from the $N N \gamma$ loop-diagrams. The corresponding $x$-dependent integrands (without the factor $\alpha / \pi$ ) are shown by the full lines in Fig. 5. Due to the suppression at low momentum-transfer, which stems from the extra-factor $l^{\alpha}$ in the vertex $V_{1}^{\mu \alpha}$, these distributions have peaks that are shifted upward to $x \simeq 0.4$.

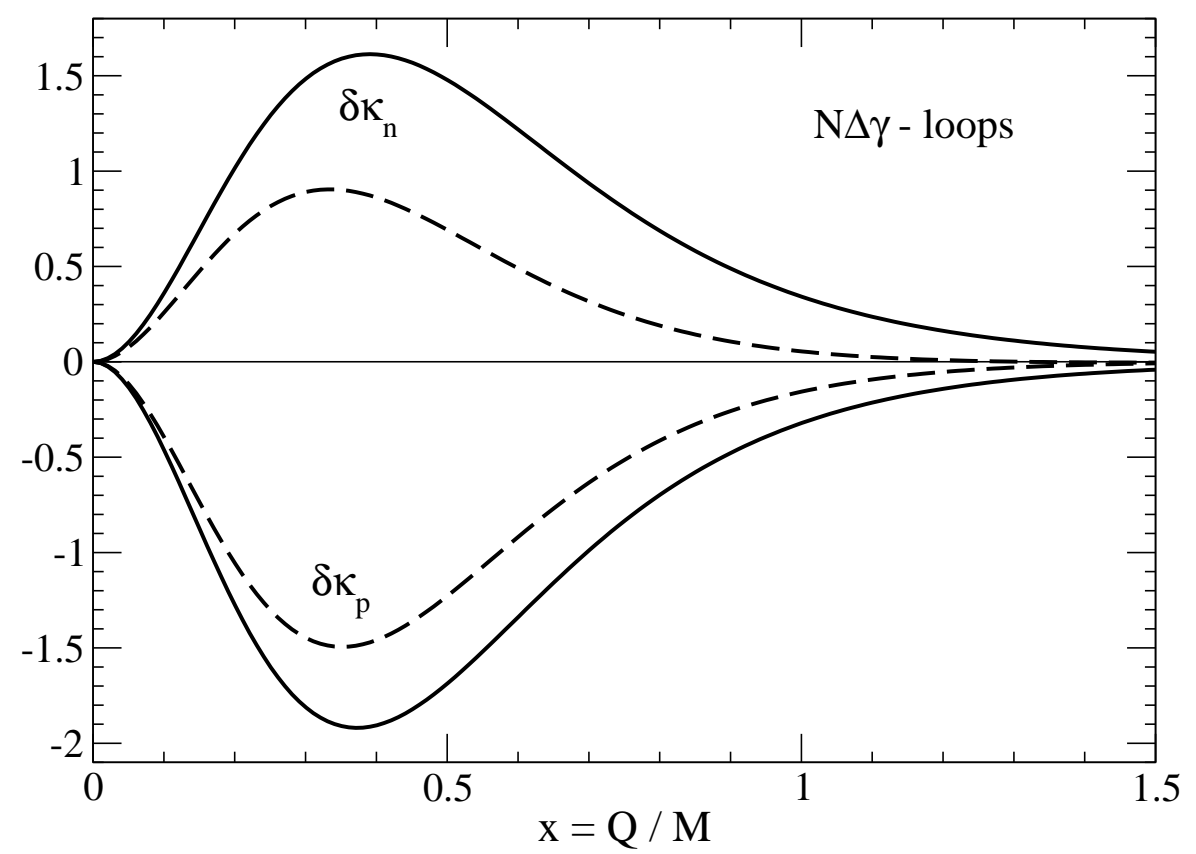

Figure 5: Integrand (without factor $\alpha / \pi$ ) for the radiative corrections from $\Delta N \gamma$-loops.

The next type for radiative correction that has to be considered is shown on the left of Fig. 6 . There, an electromagnetic self-energy insertion with an intermediate $\Delta(1232)$-isobar excitation is attached to the external nucleon lines. The nucleon wave-function renormalization factor $Z_{2}^{(\Delta)}$ 


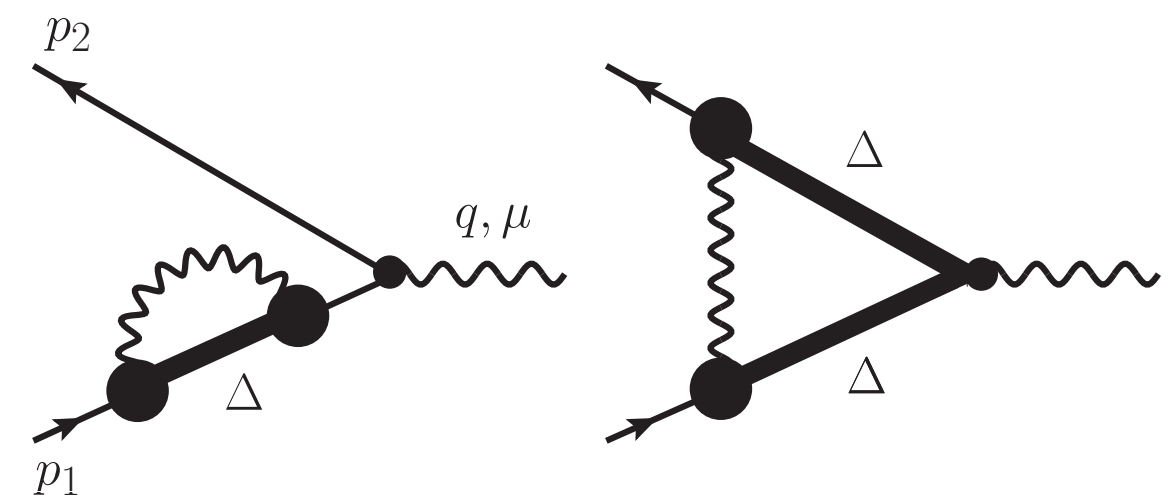

Figure 6: Left: Electromagnetic self-energy correction with virtual delta-isobar excitation on a nucleon line. Right: Photon-loop diagram with two intermediate (charged) delta-isobars.

derived from the self-energy subdiagram including a $\Delta(1232)$-isobar is given by the expression:

$$
\begin{aligned}
Z_{2}^{(\Delta)}= & \frac{\alpha \kappa^{* 2}}{\pi(12 r)^{2}} \int_{0}^{\infty} d x G_{\Delta}^{2}(x M)\left\{\frac{\left(r^{2}-1\right)^{2}}{x}\left(5+10 r^{2}+9 r^{4}\right)+12 x\left(1+2 r^{5}+r^{6}\right)\right. \\
& +6 x^{3}\left(3+4 r^{3}\right)+4 x^{5}+3 x^{7}+\sqrt{\left(r^{2}-1+x^{2}\right)^{2}+4 x^{2}}\left[\frac{1-r^{2}}{x}\left(5+10 r^{2}+9 r^{4}\right)\right. \\
& \left.\left.+x\left(7-8 r^{2}-24 r^{3}-3 r^{4}\right)+x^{3}\left(3 r^{2}-1\right)-3 x^{5}+\frac{24 r^{3} x(1+r)}{(1+r)^{2}+x^{2}}\right]\right\},
\end{aligned}
$$

and obviously it involves the square of the transition form factor $G_{\Delta}(x M)$. The corresponding contribution to the nucleon magnetic moments is $\delta \kappa=F_{2}^{(\gamma)}(0)=Z_{2}^{(\Delta)} \kappa$. Using the dipole times exponential form of $G_{\Delta}(x M)$, one finds radiative corrections to the magnetic moments with values of $\delta \kappa_{p}=1.139 \cdot 10^{-3}$ and $\delta \kappa_{n}=-1.216 \cdot 10^{-3}$. Note that these reduce the previous values from the $\Delta N \gamma$-loop to about one half in size. For further illustration we show by the full line in Fig. 7

the integrand of $Z_{2}^{(\Delta)}$ (again without the factor $\alpha / \pi$ ). The displayed distribution starts at small momentum-transfer as order $x^{3}$ and develops a peak around $x=0.5$.

\section{Photon-loop diagram with two charged delta-isobars}

Once the $\Delta(1232)$-isobar is introduced as an explicit degree of freedom, one can let it propagate further in the loop after coupling to the external photon. This mechanism requires a charged $\Delta^{+}$ and thus it is applicable only to the proton. The relevant $\Delta^{+} \Delta^{+} \gamma$ coupling is obtained directly by gauging the kinetic term of the free Rarita-Schwinger Lagrangian [9, 12], and it reads:

$$
i e\left(-\gamma^{\mu} g_{\alpha \beta}+\gamma_{\alpha} g_{\beta}^{\mu}+\gamma_{\beta} g_{\alpha}^{\mu}-\gamma_{\alpha} \gamma^{\mu} \gamma_{\beta}\right)
$$

with $\beta$ the incoming and $\alpha$ the outging (Rarita-Schwinger) index. Note that no form factor is necessary here, since this vertex is taken in the end at $t=0$. Carrying out all the (tedious) calculational steps for the right $\Delta^{+} \Delta^{+} \gamma$ loop-diagram in Fig. 6, one arrives at the following expression 


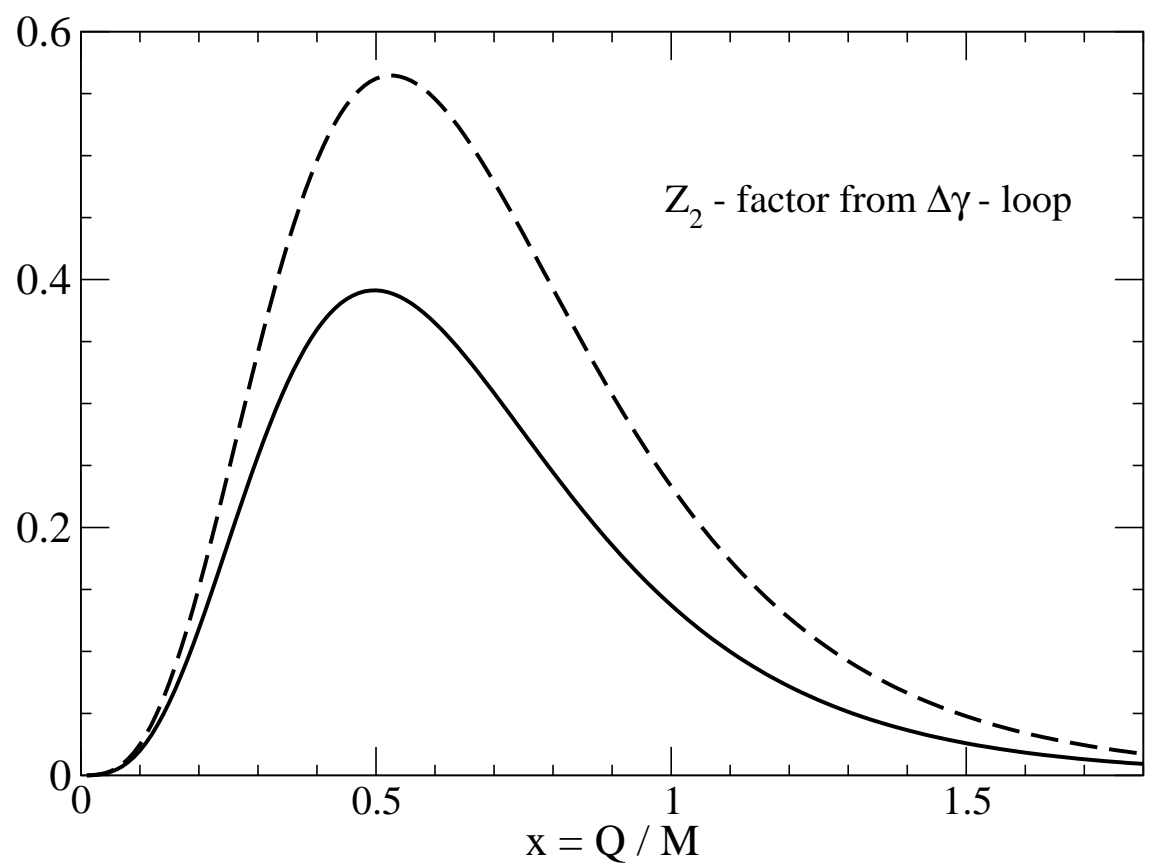

Figure 7: Integrand (without factor $\alpha / \pi$ ) for the wave-function renormalization factor $Z_{2}^{(\Delta)}$ from the $\Delta \gamma$-loop.

for the radiatively induced magnetic moment:

$$
\begin{aligned}
F_{2}^{(\gamma)}(0)= & \frac{\alpha \kappa^{* 2} c}{4 \pi(3 r)^{4}} \int_{0}^{\infty} d x G_{\Delta}^{2}(x M)\left\{\frac{\left(r^{2}-1\right)^{2}}{x}\left(1+2 r+23 r^{2}+4 r^{3}-27 r^{4}-6 r^{5}+27 r^{6}\right)\right. \\
& +2 x(1+r)\left(2-r+25 r^{2}-r^{3}+r^{4}-31 r^{5}-r^{6}+18 r^{7}\right) \\
& +6 x^{3}\left(3+r-4 r^{2}-8 r^{3}-4 r^{4}+6 r^{5}\right)+2 x^{5}\left(5 r+6 r^{2}-2\right)+x^{7}\left(4 r+9 r^{2}-1\right) \\
& +\sqrt{\left(r^{2}-1+x^{2}\right)^{2}+4 x^{2}}\left[\frac{r^{2}-1}{x}\left(27 r^{4}+6 r^{5}-27 r^{6}-1-2 r-23 r^{2}-4 r^{3}\right)\right. \\
& +x\left(3+27 r^{2}+42 r^{3}+r^{4}-40 r^{5}-9 r^{6}\right)+x^{3}\left(3-6 r-4 r^{2}+4 r^{3}+9 r^{4}\right) \\
& \left.\left.+x^{5}\left(1-4 r-9 r^{2}\right)+\frac{6 r^{4} x(1+r)(5+9 r)}{(1+r)^{2}+x^{2}}\right]\right\}
\end{aligned}
$$

where $c=1,0$ takes care that this contribution exists only for the proton. The dipole times exponential parametrization of $G_{\Delta}(x M)$ leads to a numerical value of $\delta \kappa_{p}=0.685 \cdot 10^{-3}$, which is positive and small. The corresponding integrand (without $\alpha / \pi$ ) is shown by the full line in Fig. 8 . One observes a distribution that starts near $x=0$ as order $x^{3}$ and reaches a peak at $x=0.52$.

After adding up the calculated contributions involving intermediate $\Delta(1232)$-isobars, one gets $\delta \kappa_{p}^{(\Delta)}=-0.87 \cdot 10^{-3}$ and $\delta \kappa_{n}^{(\Delta)}=1.20 \cdot 10^{-3}$. Together with the purely nucleonic loop-contributions from section 3 these yield estimates for the radiative corrections of:

$$
\delta \kappa_{p}=-4.29 \cdot 10^{-3}, \quad \delta \kappa_{n}=2.53 \cdot 10^{-3},
$$

which are still dominated by the $N N \gamma$-loop terms. As measured by the empirical magnetic moments $1+\kappa_{p}=2.793$ and $\kappa_{n}=-1.913$, these values amount to corrections of $-1.5 \%$ and $-1.3 \%$, for the proton and neutron. This is in line with the rough estimate $\alpha / 2 \pi=1.16 \%$, but opposite in sign. 


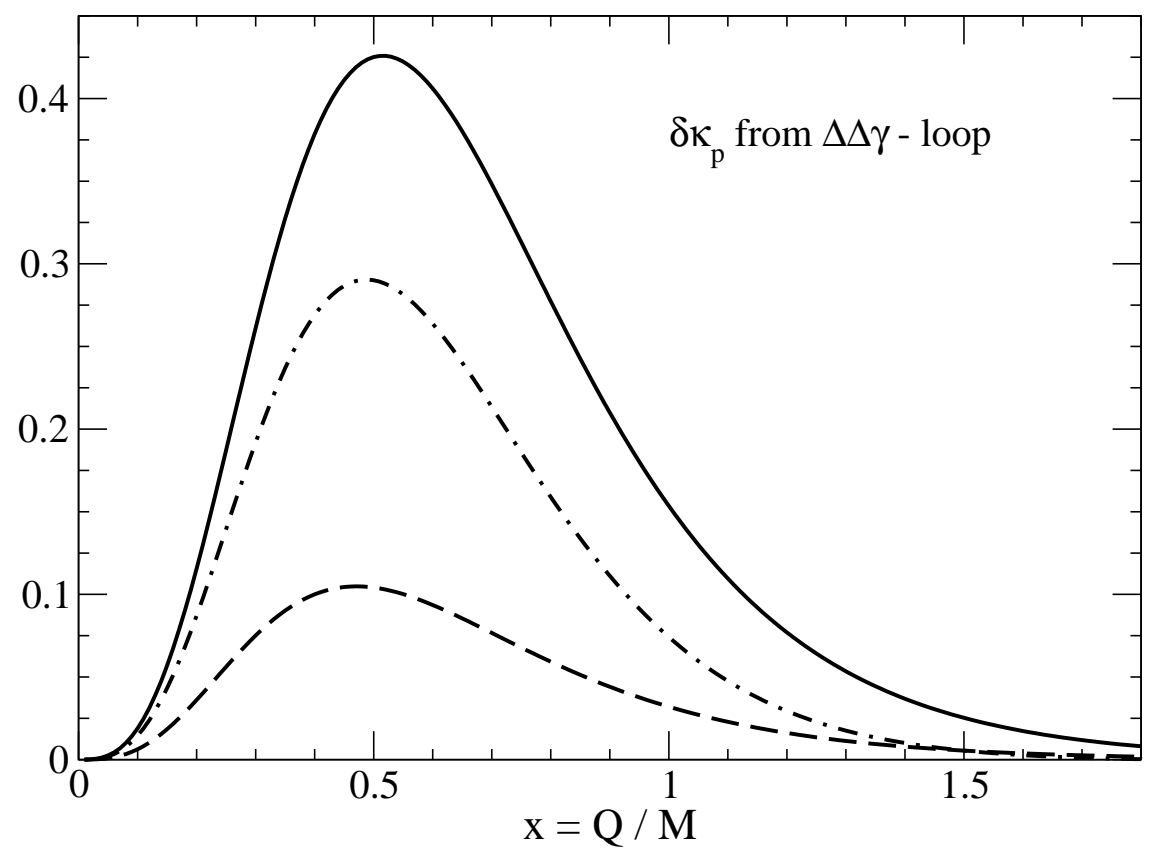

Figure 8: Integrand (without factor $\alpha / \pi$ ) for the radiative correction to the proton magnetic moment from the $\Delta^{+} \Delta^{+} \gamma$-loop.

\section{Alternative forms of spin-3/2 propagator and vertices}

The Lorentz-covariant treatment of a massive spin-3/2 particle by a Rarita-Schwinger spinor-field is affected by various problems concerning the presence of spurious spin- $1 / 2$ degrees of freedom. For this reason, it has been proposed in recent works [7, 10, 11] to use for the $\Delta$-isobar a modified propagator of the form:

$$
\frac{i}{3} \frac{\gamma \cdot P+M_{\Delta}}{M_{\Delta}^{2}-P^{2}}\left[3 g_{\alpha \beta}-\gamma_{\alpha} \gamma_{\beta}-\frac{1}{P^{2}}\left(\gamma \cdot P \gamma_{\alpha} P_{\beta}+P_{\alpha} \gamma_{\beta} \gamma \cdot P\right)\right],
$$

which includes a suitably constructed projector on spin-3/2. The use of such a $\Delta$-isobar propagator introduces into our calculation of photon-loop diagrams a further denominator $P^{2}$ that depends on the loop-momentum $l$. When combining it with the $N \Delta \gamma$-vertex $V_{1}^{\mu \alpha}$ in eq.(12) one gets, as a result of the angular integration $\int_{-1}^{1} d z \sqrt{1-z^{2}}$, weight-functions that have a discontinuity at $x=1$. This feature is exemplified in the appendix for the electromagnetic mass shift $\delta M$. We consider the appearance of discontinuities as an indication for an inconsistent treatment and work with an alternative form of the $\Delta N \gamma$-vertex taken from refs. [7, 10, 11]:

$$
V_{2}^{\mu \alpha}=\frac{i e \kappa^{*}}{\sqrt{6 M^{3} M_{\Delta}}}\left(g^{\mu \alpha} \gamma \cdot P \gamma \cdot l-P^{\mu} \gamma^{\alpha} \gamma \cdot l-\gamma^{\mu} \gamma^{\alpha} P \cdot l+\gamma^{\mu} l^{\alpha} \gamma \cdot P\right) \gamma_{5} G_{\Delta}\left(\sqrt{-l^{2}}\right)
$$

It fulfils the relations $l_{\mu} V_{2}^{\mu \alpha}=0$ (gauge-invariance) and $P_{\alpha} V_{2}^{\mu \alpha}=0$ (orthogonality). The prefactor with square-roots of masses in the denominator has been chosen such that the residue of the spinindependent (real) forward Compton-amplitude $N \gamma \rightarrow \Delta \rightarrow N \gamma$ at the $\Delta$ (1232)-pole remains constant. For the first vertex $V_{1}^{\mu \alpha}$ this residue is proportional to $3+r^{-2}=3.581$, while for the second vertex $V_{2}^{\mu \alpha}$ it is (in the same way) proportional to $r+3 r^{-1}=3.599$, and both numbers agree very well with each other. It should be noted that the vertex $V_{2}^{\mu \alpha}$ in eq.(21) is just a minimal version with a single coupling constant $\kappa^{*}$ and extensions with further parameters have been proposed in the literature [7, 11]. 


\begin{tabular}{|c|ccc|}
\hline form factor & dipole & DRA & Ina \\
\hline $10^{3} \cdot \delta \kappa_{p}$ & -1.911 & -1.900 & -1.896 \\
$10^{3} \cdot \delta \kappa_{n}$ & 1.112 & 1.063 & 1.055 \\
\hline
\end{tabular}

Tab.3: Radiative corrections to the proton and neutron magnetic moments arising from $\Delta N \gamma$ triangle-diagrams using with the second vertex.

We turn now to the reevaluation of the triangular photon-loop diagrams in Fig. 4 with the new $\Delta N \gamma$-vertex $V_{2}^{\mu \alpha}$. Independent of the choice of the $\Delta$-isobar propagator (eq.(13) or eq.(20)), this contribution to the radiatively induced magnetic moment reads:

$$
\begin{aligned}
F_{2}^{(\gamma)}(0)= & \frac{\alpha \kappa^{* 2}}{54 \pi r} \int_{0}^{\infty} d x G_{\Delta}(x M)\left\{F _ { 1 } ( x M ) \left[-\frac{3+r^{2}}{x}\left(r^{2}-1\right)^{2}-12 x\left(1+r^{3}+x^{2} r\right)\right.\right. \\
& -4 x^{5}-\frac{x^{2}}{r^{2}-1} \sqrt{4+x^{2}}\left(4+12 r+10 x^{2}+3 x^{4}\right)+\sqrt{\left(r^{2}-1+x^{2}\right)^{2}+4 x^{2}} \\
& \left.\times\left(\frac{3+r^{2}}{x}\left(r^{2}-1\right)+\frac{x}{r^{2}-1}\left(9-4 r^{2}+12 r^{3}-r^{4}+x^{2}\left(9+r^{2}\right)+3 x^{4}\right)\right)\right] \\
& +F_{2}(x M)\left[-\frac{\left(r^{2}-1\right)^{2}}{2 x}\left(3+r^{2}+4 r^{3}\right)-x\left(6+2 r^{2}+9 r^{3}+2 r^{4}+3 r^{5}+2 r^{6}\right)\right. \\
& -6 x^{3}\left(3+r+2 r^{2}+r^{4}\right)+x^{5}\left(r-14-6 r^{2}\right)-2 x^{7}+\frac{x^{2}}{r^{2}-1} \sqrt{4+x^{2}}\left(x^{2}(r-13)\right. \\
& \left.-8(1+r)-\frac{7 x^{4}}{2}\right)+\frac{1}{2} \sqrt{\left(r^{2}-1+x^{2}\right)^{2}+4 x^{2}}\left(\frac{r^{2}-1}{x}\left(3+r^{2}+4 r^{3}\right)+\frac{x}{r^{2}-1}\right. \\
& \left.\left.\left.\times\left(9+14 r^{3}+3 r^{4}+2 r^{5}+4 r^{6}+x^{2}\left(9+9 r^{2}-2 r^{3}+8 r^{4}\right)+x^{4}\left(3+4 r^{2}\right)\right)\right)\right]\right\}
\end{aligned}
$$

with the mass ratio $r=M_{\Delta} / M=1.312$. One notices that only the part of the Rarita-Schwinger propagator proportional to $3 g_{\alpha \beta}-\gamma_{\alpha} \gamma_{\beta}$ has an influence on the result and therefore the additional loop-factor $1 / P^{2}$ cannot generate a discontinuity in the weight-functions at $x=1$.

Table 3 gives the corresponding numerical values of $\delta \kappa_{p}$ and $\delta \kappa_{p}$ for the three choices (dipole, DRA, Ina) of the nucleon vector form factors $F_{1,2}(x M)$. In comparison to the first $\Delta N \gamma$-vertex, one obtains singificantly reduced values of $\delta \kappa_{p} \simeq-1.9 \cdot 10^{-3}$ and $\delta \kappa_{n} \simeq 1.1 \cdot 10^{-3}$. This reduction becomes also obvious from the underlying integrands which are shown by the dashed lines in Fig. 5 . The differences between the full and dashed lines are suggestive of spurious spin-1/2 degrees of freedom in the Rarita-Schwinger spinor.

Next we have to reevaluate with the new vertex $V_{2}^{\mu \alpha}$ the wave-function renormalization factor $Z_{2}^{(\Delta)}$ arising from the self-energy subdiagram in Fig. 6 . Independent of the choice of the $\Delta$-isobar propagator this quantity is now given by the expression:

$$
\begin{aligned}
Z_{2}^{(\Delta)}= & \frac{\alpha \kappa^{* 2}}{48 \pi r} \int_{0}^{\infty} d x G_{\Delta}^{2}(x M)\left\{\frac{\left(r^{2}-1\right)^{2}}{x}\left(5+2 r^{2}+r^{4}\right)+4 x\left(3-r^{2}+2 r^{5}\right)+2 x^{3}\left(3+4 r^{3}\right)\right. \\
& +4 x^{5}\left(1+r^{2}\right)+3 x^{7}+\sqrt{\left(r^{2}-1+x^{2}\right)^{2}+4 x^{2}}\left[\frac{1-r^{2}}{x}\left(5+2 r^{2}+r^{4}\right)\right. \\
& \left.\left.+x\left(7-4 r^{2}-8 r^{3}+r^{4}\right)-x^{3}\left(1+r^{2}\right)-3 x^{5}+\frac{8 r^{3} x(1+r)}{(1+r)^{2}+x^{2}}\right]\right\}
\end{aligned}
$$

The corresponding integrand (without the factor $\alpha / \pi$ ) is shown by the dashed line in Fig. 7. The apparent enhancement by a factor of about 1.5 translates into more sizeable corrections of opposite sign: $\delta \kappa_{p}=1.732 \cdot 10^{-3}$ and $\delta \kappa_{n}=-1.848 \cdot 10^{-3}$. 
Finally, we have to reevaluate the $\Delta^{+} \Delta^{+} \gamma$ loop-diagram in Fig.6 with the new vertex $V_{2}^{\mu \alpha}$. In this case the result for the radiatively induced magnetic moment of the proton depends on the choice the $\Delta$-isobar propagator. Using the not spin-3/2 projected version in eq.(13), one gets the expression:

$$
\begin{aligned}
F_{2}^{(\gamma)}(0)= & \frac{\alpha \kappa^{* 2} c}{\pi(18 r)^{2}} \int_{0}^{\infty} d x G_{\Delta}^{2}(x M)\left\{\frac{r}{x}\left(r^{2}-1\right)^{2}\left(23-8 r^{2}+9 r^{4}\right)+2 x^{5}\left(18 r^{3}-9 r^{2}+26 r-24\right)\right. \\
& +2 x\left(8+24 r+15 r^{2}-45 r^{4}-12 r^{5}+34 r^{6}\right)+6 x^{3}\left(11 r^{4}-8 r^{2}-16\right)+x^{7}(27 r-16) \\
& +\sqrt{\left(r^{2}-1+x^{2}\right)^{2}+4 x^{2}}\left[\frac{r}{x}\left(1-r^{2}\right)\left(23-8 r^{2}+9 r^{4}\right)+x^{3}\left(32-25 r+2 r^{2}-9 r^{3}\right)\right. \\
& \left.\left.+x\left(16+25 r+46 r^{2}-20 r^{3}-68 r^{4}+9 r^{5}\right)+x^{5}(16-27 r)+\frac{6 r^{3} x(1+r)(5+9 r)}{(1+r)^{2}+x^{2}}\right]\right\}
\end{aligned}
$$

which gives the numerical value $\delta \kappa_{p}=0.415 \cdot 10^{-3}$. The corresponding integrand is shown by the dashed-dotted line in Fig. 8. On the other hand the spin-3/2 projected version in eq.(20) leads to a somewhat different expression:

$$
\begin{aligned}
F_{2}^{(\gamma)}(0)= & \frac{\alpha \kappa^{* 2} c}{324 \pi r} \int_{0}^{\infty} d x G_{\Delta}^{2}(x M)\left\{\frac{\left(r^{2}-1\right)^{2}}{x}\left(3+8 r^{2}+13 r^{4}\right)+6 x^{3}\left(4 r+23 r^{3}\right)\right. \\
& +2 x\left(8+9 r-45 r^{3}+48 r^{5}+4 r^{6}\right)+2 x^{5}\left(18+21 r+14 r^{2}\right)+23 x^{7} \\
& +\sqrt{\left(r^{2}-1+x^{2}\right)^{2}+4 x^{2}}\left[\frac{1-r^{2}}{x}\left(3+8 r^{2}+13 r^{4}\right)-x^{3}\left(13+42 r+5 r^{2}\right)\right. \\
& \left.\left.+x\left(13+18 r-28 r^{2}-96 r^{3}+5 r^{4}\right)-23 x^{5}+\frac{6 r^{2} x(1+r)(5+9 r)}{(1+r)^{2}+x^{2}}\right]\right\},
\end{aligned}
$$

which yields a significantly smaller numerical value of $\delta \kappa_{p}=0.163 \cdot 10^{-3}$. This feature is also indicated by the dashed line in Fig. 8 for the respective integrand. It is interesting to note that in the last case only the quasi-Dirac part $-i e g_{\alpha \beta} \gamma^{\mu}$ of the $\Delta^{+} \Delta^{+} \gamma$-coupling has contributed. For the spin-3/2 projected vertex and propagator the terms involving intermediate $\Delta(1232)$-isobars sum up to $\delta \kappa_{p}^{(\Delta)}=0.00 \cdot 10^{-3}$ and $\delta \kappa_{n}^{(\Delta)}=-0.79 \cdot 10^{-3}$. Together with the purely nucleonic loop-contributions from section 3 these yield then estimates for the radiative corrections of:

$$
\delta \kappa_{p}=-3.42 \cdot 10^{-3}, \quad \delta \kappa_{n}=0.54 \cdot 10^{-3},
$$

which are (unfortunately) quite different from the first estimate given in eq.(19).

\section{Summary and conclusions}

In this work we have estimated the radiative corrections of order $\alpha / \pi$ to the magnetic moments of the proton and neutron. The Schwinger calculation for a structureless lepton has been adapted to extended nucleons by including phenomenological vector form factors. The resulting expression for $F_{2}^{(\gamma)}(0)$ is infrared-finite and gauge-invariant and yields numerical values of $\delta \kappa_{p}=-3.42 \cdot 10^{-3}$ and $\delta \kappa_{n}=1.34 \cdot 10^{-3}$, using updated parametrizations for the nucleon form factors $F_{1,2}(Q)$. Moreover, we have also studied the effects of photon-loops involving the low-lying $\Delta(1232)$-resonance. For the pertinent calculation a Lorentz-covariant treatment of massive spin-3/2 particles is mandatory. The two (minimal) versions of the $\Delta N \gamma$-vertex amended by a phenomenological transition form factor $G_{\Delta}(Q)$, that we have examined in combination with costumary form of the spin-3/2 propagator, 
yield radiative corrections of $\delta \kappa_{p}^{(\Delta)}=(-0.9,0.0) \cdot 10^{-3}$ and $\delta \kappa_{n}^{(\Delta)}=(1.2,-0.8) \cdot 10^{-3}$, respectively. The differences are quite substantial and therefore one may hope that an extended form of the $\Delta \rightarrow N \gamma$ transition amplitude (with further coupling constants and off-shell parameters) will lead to radiatively induced nucleon magnetic moments that are more consistent for conventional and spin-3/2 projected vertices and propagators.

\section{Acknowledgements}

I thank A.F. Rey-Fernandez for participation in this project during his bachelor thesis. Ina Lorenz has provided valuable information on updated nucleon form factor parametrizations and on approaches to the spin-3/2 delta-isobar.

\section{Appendix: Electromagnetic mass-shifts}

In this appendix the expressions for electromagnetic mass-shifts of the nucleon are given. The calculation of the self-energy subdiagram on the right of Fig. 2 with a $N \gamma$-loop leads to the following gauge-invariant (or $\xi$-independent) result:

$$
\begin{aligned}
\delta M= & \frac{\alpha M}{4 \pi} \int_{0}^{\infty} d x\left\{\left[\left(2-x^{2}\right) \sqrt{4+x^{2}}+x^{3}\right] F_{1}^{2}(x M)+3 x^{2}\left(x-\sqrt{4+x^{2}}\right)\right. \\
& \left.\times F_{1}(x M) F_{2}(x M)+\frac{x^{2}}{8}\left[\left(x^{2}-8\right) \sqrt{4+x^{2}}+6 x-x^{3}\right] F_{2}^{2}(x M)\right\},
\end{aligned}
$$

with $F_{1,2}(x M)$ the Dirac and Pauli form factors. By evaluating this one-parameter integral for the three choices (dipole, DRA, Ina) of nucleon form factors, one finds for the electromagnetic proton-neutron mass difference $\delta M_{p-n}=(0.795,0.757,0.782) \mathrm{MeV}$, respectively.

Next, we consider the self-energy diagram with a $\Delta \gamma$-loop. Using the first vertex $V_{1}^{\mu \alpha}$ in eq.(12) and the conventional Rarita-Schwinger propagator in eq.(13), one the obtains the expression:

$$
\begin{aligned}
\delta M= & \frac{\alpha M \kappa^{* 2}}{\pi(12 r)^{2}} \int_{0}^{\infty} d x G_{\Delta}^{2}(x M)\left\{\frac{1+3 r^{2}}{x}\left(1-r^{2}\right)^{3}-x^{7}+6 x^{3}\left(3-2 r^{3}\right)\right. \\
& -4 x^{5}+4 x\left(1-r^{2}\right)\left(1+r^{2}+3 r^{3}+r^{4}\right)+\sqrt{\left(r^{2}-1+x^{2}\right)^{2}+4 x^{2}} \\
& \left.\times\left[\frac{1+3 r^{2}}{x}\left(r^{2}-1\right)^{2}+x\left(3+12 r^{3}+r^{4}\right)+x^{3}\left(3-r^{2}\right)+x^{5}\right]\right\},
\end{aligned}
$$

with $G_{\Delta}(x M)$ the phenomenological $\Delta \rightarrow N \gamma$ transition form factor. The dipole times exponential function yields a mass-shift of $\delta M=0.462 \mathrm{MeV}$, equal for the proton and neutron. When using the second vertex $V_{2}^{\mu \alpha}$ in eq.(21) the result for $\delta M$ is actually independent of the choice of the $\Delta$-isobar propagator, and it reads:

$$
\begin{aligned}
\delta M= & \frac{\alpha M \kappa^{* 2}}{144 \pi r} \int_{0}^{\infty} d x G_{\Delta}^{2}(x M)\left\{\frac{3+r^{2}}{x}\left(1-r^{2}\right)^{3}+12 x\left(1-r^{2}\right)\left(1+r^{3}\right)\right. \\
& +6 x^{3}\left(3-4 r-2 r^{3}\right)-4 x^{5}\left(3+r^{2}\right)-3 x^{7}+\sqrt{\left(r^{2}-1+x^{2}\right)^{2}+4 x^{2}} \\
& \left.\times\left[\frac{3+r^{2}}{x}\left(r^{2}-1\right)^{2}+x\left(9-4 r^{2}+12 r^{3}-r^{4}\right)+x^{3}\left(9+r^{2}\right)+3 x^{5}\right]\right\} .
\end{aligned}
$$

The corresponding numerical value $\delta M=0.258 \mathrm{MeV}$ is reduced by a factor 0.56 compared to the first case. For the sake of completeness we present also the expression for $\delta M$ obtained by 
combining the first vertex $V_{1}^{\mu \alpha}$ with the spin-3/2 projected propagator in eq.(20). It reads:

$$
\begin{aligned}
\delta M= & \frac{\alpha M \kappa^{* 2}}{144 \pi} \int_{0}^{\infty} d x G_{\Delta}^{2}(x M)\left\{\operatorname{sign}(1-x) \frac{\left(1+x^{2}\right)^{4}}{r^{2} x}+\frac{r^{2}}{x}\left(8 r^{2}-6-3 r^{4}\right)\right. \\
& +4 r x\left(3-3 r^{2}-r^{3}-3 x^{2}\right)+\frac{1}{r^{2}} \sqrt{\left(r^{2}-1+x^{2}\right)^{2}+4 x^{2}} \\
& \left.\times\left[\frac{1+3 r^{2}}{x}\left(r^{2}-1\right)^{2}+x\left(3+12 r^{3}+r^{4}\right)+x^{3}\left(3-r^{2}\right)+x^{5}\right]\right\},
\end{aligned}
$$

and includes a (peculiar) discontinuous part which stems from the angular integral:

$$
\frac{4}{\pi} \int_{-1}^{1} d z \frac{x^{2} \sqrt{1-z^{2}}}{x^{2}-1+2 i x z}=1-x^{2}-\left(1+x^{2}\right) \operatorname{sign}(1-x) .
$$

The numerically computed mass-shift $\delta M=0.348 \mathrm{MeV}$ lies just in between the two previous values.

\section{References}

[1] Particle Data Group, Chin. Phys. C38, 090001 (2014).

[2] F. Jegerlehner, "The Anomalous Magnetic Moment of the Muon", Springer Tracts in Modern Physics 226, Springer Verlag, Berlin Heidelberg (2008); chapter 3.5.

[3] A.W. Thomas and W. Weise, "The Structure of the Nucleon", WILEY-VCH Verlag, Berlin (2001).

[4] P. Mergell, Ulf-G. Meißner, D. Drechsel, Nucl. Phys. A596, 367 (1996).

[5] I.T. Lorenz, H.W. Hammer, Ulf-G. Meißner, Eur. Phys. J. A48, 151 (2012).

[6] I.T. Lorenz and Ulf-G. Meißner, Phys. Lett. B737, 57 (2014).

[7] I.T. Lorenz, Ulf-G. Meißner, H.W. Hammer, Y.B. Dong, Phys. Rev. D91, 014023 (2015).

[8] Ina Lorenz, private communications.

[9] M. Benmerrouche, R.M. Davidson, N.C. Mukhopadhyay, Phys. Rev. C39, 2339 (1989); and refs. therein.

[10] S. Kondratyuk and O. Scholten, Phys. Rev. C64, 024005 (2001).

[11] V. Pascalutsa, M. Vanderhaeghen, S.N. Yang, Phys. Reports 437, 125 (2007); and refs. therein.

[12] V. Bernard, N. Kaiser, Ulf-G. Meißner, Int. J. Mod. Phys. E4, 193 (1995); appendix E.

[13] V. Burkert and Z. Li, Phys. Rev. D47, 46 (1993); and refs. therein. 